\title{
Optimization of thick-walled shells based on solutions of inverse problems of the elastic theory for inhomogeneous bodies
}

\author{
V. I. Andreev \\ Department of Strength of Materials, \\ Moscow State University of Civil Engineering, Russia
}

\begin{abstract}
An inverse problem of elastic theory for inhomogeneous bodies is normally used for identification of the dependencies of a material's mechanical properties of coordinates where the stress state of the body will be specified. It is known that in thick-walled cylindrical or spherical shells under internal or external pressure the highest stresses are close to the inner surface of the shell. Several solutions of inverse problems (depending on the elastic modulus along the radius at which the equivalent stress in the shell will be constant) are obtained in this paper with the use of conventional strength theories. Corresponding shells could be called equal stress shells. If an investigator changes the elastic modulus of the material its mechanical properties change as well. It is shown that for some materials the investigator can create a model of an equal strength shell with an equivalent stress at each point, which is equal to the strength of the material. This paper is devoted to creating multi-layered shells in which the elastic modulus in each layer is determined by the results of solving inverse problems.

Keywords: elastic theory, inverse problem, thick-walled shells, inhomogeneous bodies, stress state, strength, equivalent stress, multilayer shells, maximum shear theory, maximum-strain-energy theory.
\end{abstract}

\section{Introduction}

The aim is to develop models of thick-walled shells which are close to equal strength. The modulus of elasticity of the material, which depends on the radius at which the corresponding equivalent stress will be constant at all points of the shell [1], is determined with the use of solutions of inverse elasticity problems 
for inhomogeneous bodies for various strength theories. Analytical and numerical methods are used for the solution of inverse problems. A special socalled coefficient of efficiency of design $\beta$ (the ratio of the maximum limiting pressure in an inhomogeneous shell (with the variable modulus of elasticity) to the corresponding pressure in a homogeneous shell) is introduced in this paper. We use the criterion of strength as given by Batrakov [2] and the model of Karpenko [5] for analysis of the corresponding shells. The coefficient $\beta$ for different compositions of concrete and different loading conditions is normally within the interval 1.5-2.6.

\section{Ideas}

Fig. 1 shows a cross section of a thick-walled cylindrical shell, loaded inside with constant pressure $p_{a}$ (fig. 1,a). For a homogeneous material (shown by dotted lines in fig. $1, b)$ the stress $\sigma_{\theta}$ reaches a maximum near the inner contour of the cylinder (fig. 1,c), and for a heterogeneous material (solid line), where $E=E(r)$, the diagram $\sigma_{\theta}$ aligns more closely to the constant value. The idea of the method of optimizing in the strength of the thick-walled shells consists in the creation of such shells from heterogeneous materials. For this purpose we solve the inverse problem of elasticity of inhomogeneous bodies for the respective shell. The essence of the inverse problem is the following. Assuming that for all points of the shell the equivalent stress $\sigma_{0}$, corresponding to a particular theory of strength, is constant, we can define the proper function $E(r)$. This shell is called an equal-stress shell.

a)

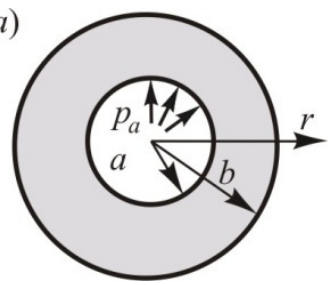

b)

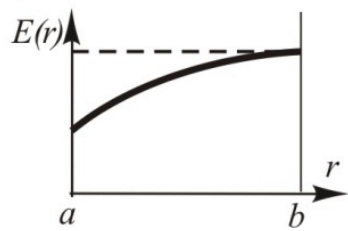

c)

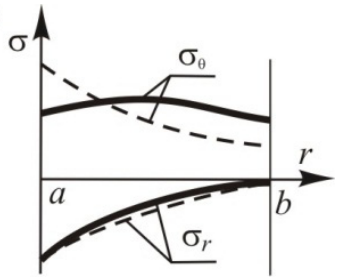

Figure 1: $\quad$ Stress state in a thick-walled cylinder under the action of internal pressure. — nonhomogeneous material, - - - homogeneous material.

\section{The solutions of inverse problems for a thick-walled cylinder for two classical theories of strength}

Below for example are the solutions of inverse problems for maximum shear theory and maximum-strain-energy theory. 


\subsection{The basic equations}

The solution of the direct problem of the theory of elasticity of inhomogeneous bodies in cylindrical coordinates with axial symmetry is reduced to a differential equation [1]

$$
\sigma_{r}^{\prime \prime}+\left(\frac{3}{r}-\frac{E^{\prime}}{E}\right) \sigma_{r}^{\prime}-\frac{1-v}{r} \cdot \frac{E^{\prime}}{E} \sigma_{r}=0
$$

Here the modulus of elasticity $E=E(r)$. The essence of the direct problem is to determine the stress state at a known function of $E(r)$. As mentioned above, the essence of solving the inverse problem is to determine the function $E(r)$ in which the equivalent stress $\sigma_{0}$ (for each theory of strength its own) will be constant throughout the structure. We consider a thick-walled cylindrical shell, where the inner radius is equal to $a$ and the external radius is equal to $b$, with the loaded constant internal $\left(p_{a}\right)$ and external $\left(p_{b}\right)$ pressures. In this case, the boundary conditions have the form:

$$
r=a, \quad \sigma_{r}=-p_{a} ; \quad r=b, \quad \sigma_{r}=-p_{b} .
$$

\subsection{Maximum shear theory}

\subsubsection{Option 1}

If circumferential stresses $\sigma_{\theta}>0$ the principal stresses are defined as follows: $\sigma_{1}=\sigma_{\theta}, \sigma_{2}=\sigma_{z}=v\left(\sigma_{r}+\sigma_{\theta}\right)$ and $\sigma_{3}=\sigma_{r}$. In view of this condition the equal-stress state is written in the form $\sigma_{\theta}-\sigma_{r}=\sigma_{0}=$ const. Substituting this into the equilibrium equation

$$
\frac{d \sigma_{r}}{d r}+\frac{\sigma_{r}-\sigma_{\theta}}{r}=0
$$

we obtain

$$
\sigma_{r}^{\prime}=\frac{\sigma_{0}}{r}
$$

The solution of this differential equation is a function

$$
\sigma_{r}=\sigma_{0} \ln r+A
$$

From the boundary conditions (2) we find the constants $A$ and $\sigma_{0}$ :

$$
A=\frac{p_{b} \ln a-p_{a} \ln b}{\ln b-\ln a} ; \sigma_{0}=\frac{p_{a}-p_{b}}{\ln b-\ln a}
$$

From eqn (3) we obtain an expression for $\sigma_{\theta}$ :

$$
\sigma_{\theta}=\sigma_{0}(1+\ln r)+A
$$

Substituting (5) in eqn (1), we obtain the equation for modulus $E(r)$ :

$$
E^{\prime}-\frac{2 \sigma_{0}}{r\left[\sigma_{0}(1+k \cdot \ln r)+A \cdot k\right]} \cdot E=0
$$

where $k=(1-2 v) /(1-v)$. 
After separation of variables in eqn (8), integrating and using the initial condition ( $\left.r=a ; E=E_{0}\right)$, we obtain the relationship $E(r)$ :

$$
E(r)=E_{0}\left[\frac{\left(p_{a}-p_{b}\right)(1+k \ln r)+k\left(p_{b} \ln a-p_{a} \ln b\right)}{\left(p_{a}-p_{b}\right)(1+k \ln a)+k\left(p_{b} \ln a-p_{a} \ln b\right)}\right]^{\frac{2}{k}} .
$$

\subsubsection{Option 2}

Under the action in the circumferential direction $\sigma_{\theta}<0$ the principal stresses are equal: $\sigma_{1}=\sigma_{r}, \quad \sigma_{2}=\sigma_{z}=v\left(\sigma_{r}+\sigma_{\theta}\right)$ and $\sigma_{3}=\sigma_{\theta}$. With this in mind the equal-stress condition takes the form $\sigma_{r}-\sigma_{\theta}=\sigma_{0}=$ const. In this case we obtain a solution that coincides with the solution given in Option 1.

\subsubsection{Option 3}

Under the action in the circumferential direction $\sigma_{\theta}<0$ the principal stresses can be determined also as follows: $\sigma_{1}=\sigma_{z}=v\left(\sigma_{r}+\sigma_{\theta}\right), \sigma_{2}=\sigma_{r}$ and $\sigma_{3}=\sigma_{\theta}$. With this in mind the equal-stress condition after some changing is the form: $\left[(1-k) \sigma_{r}-\sigma_{\theta}\right] /(2-k)=\sigma_{0}=$ const . Expressing from this equality $\sigma_{\theta}$ and substituting it into the equilibrium eqn (3), we obtain

$$
\sigma_{r}^{\prime}+k \frac{\sigma_{r}}{r}=-(2-k) \cdot \frac{\sigma_{0}}{r}
$$

The solution of this differential equation is the function:

$$
\sigma_{r}=\frac{A \cdot r^{-k}-(2-k) \sigma_{0}}{k}
$$

We can define constants $A$ and $\sigma_{0}$ using the boundary conditions (2):

$$
A=k \cdot \frac{p_{a}-p_{b}}{b^{-k}-a^{-k}} ; \quad \sigma_{0}=\frac{k}{2-k} \cdot \frac{p_{a} b^{-k}-p_{b} a^{-k}}{b^{-k}-a^{-k}}
$$

Substituting the stress (11) into the resolving eqn (1) we obtain a homogeneous linear differential equation for the distribution of the modulus of elasticity:

$$
E^{\prime}-\frac{A}{\sigma_{0}} \cdot r^{-(k+1)} \cdot E=0
$$

After integrating eqn (13), with the condition ( $\left.r=a ; E=E_{0}\right)$ we obtain the dependence $E(r)$ :

$$
E(r)=E_{0} \exp \left[-\frac{A}{k \sigma_{0}} \cdot\left(r^{-k}-a^{-k}\right)\right]
$$

In fig. 2 diagrams of the dependence $E(r)$ are presented that are calculated using the values: $v_{1}=0.1, v_{2}=0.25, v_{3}=0.4, b / a=2, p_{a}=6 \mathrm{MPa}, p_{b}=12$ MPa. 
For values of Poisson's ratio $v_{1}=0.1$ and $v_{2}=0.25$ the dependence $E(r)$ is determined by solving the optimization problem for a cylinder corresponding to option 3 , since the stress relation to this variant is $\sigma_{z}>\sigma_{r}>\sigma_{\theta}$. However, for the value $v_{3}=0.4$ the solution of the optimization problem for a cylinder corresponds to option 1 , since the stress relation to that decision has $\sigma_{r}>\sigma_{z}>\sigma_{\theta}$, taking into account amendments to the sign of the equivalent stress. As follows from fig. 2 the influence of Poisson's ratio for the distribution function $E(r)$ is significant. In fig. 3 as an example the distribution of stresses in the inhomogeneous (equal-stress) cylinder in the case $v_{2}=0.25$ is shown, as well as that in a homogeneous cylinder with the same sizes and loads.

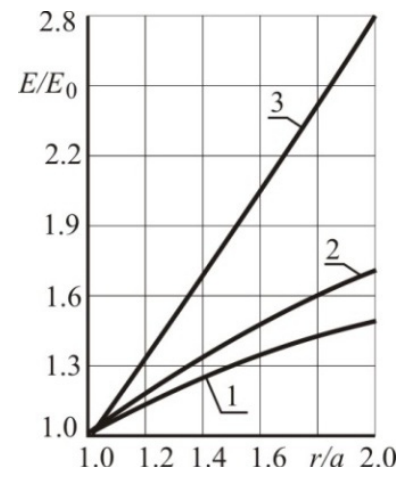

Figure 2: Distribution of the modulus of elasticity in the equal-stress cylinder. $1-v=0.1,2$ $-v=0.25,3-v=0.4$

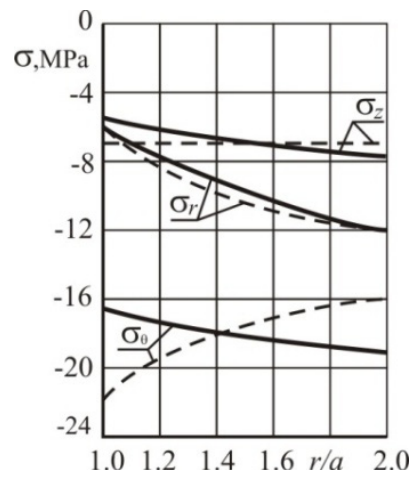

Figure 3: Stress diagrams in the equal-stress cylinder. - nonhomogeneous material, - - homogeneous material

Once again we will underline that the equivalent stress $\sigma_{0}$ at all points of the cylinder is the same. Thus, we have found the model of the equal-stress structure. However, such a cylinder is not equal-strength. To learn how to get the model of the equal-strength cylinder we will discuss things further.

\subsection{Maximum-strain-energy theory}

The fourth theory of strength in the coordinate system $\sigma_{1}, \sigma_{2}$ and $\sigma_{3}$ represents the surface of a circular cylinder

$$
\sigma_{1}^{2}+\sigma_{2}^{2}+\sigma_{3}^{2}-\left(\sigma_{1} \sigma_{2}+\sigma_{2} \sigma_{3}+\sigma_{3} \sigma_{1}\right)=\sigma_{0}^{2}
$$

So depending on what type of structure (disk or cylinder) is calculated, the configuration of the surface determining the strength of the material of the 
structure will vary. Therefore, to best present the whole picture it is useful to consider separately the calculation of the disk and the cylinder. Below we consider the construction of the equal-stress model of the cylinder.

The principal stresses in this case are defined by: $\sigma_{1}=\sigma_{z}=v\left(\sigma_{r}+\sigma_{\theta}\right)$, $\sigma_{2}=\sigma_{r}$ and $\sigma_{3}=\sigma_{\theta}$. In view of this condition of equal-strength (15) can be rewritten as

$$
\left(1-v+v^{2}\right) \sigma_{r}^{2}-\left(1+2 v-2 v^{2}\right) \sigma_{r} \sigma_{\theta}+\left(1-v+v^{2}\right) \sigma_{\theta}^{2}=\sigma_{0}^{2}=\text { const }
$$

The eqn (16) describes an ellipse, so the solution is a necessary search using parametric expressions for the stresses:

$$
\sigma_{r}=\sigma_{0}\left(\frac{2-k}{k} \sin \varphi-\frac{1}{\sqrt{3}} \cos \varphi\right), \quad \sigma_{\theta}=\sigma_{0}\left(\frac{2-k}{k} \sin \varphi+\frac{1}{\sqrt{3}} \cos \varphi\right)
$$

Substituting into the equilibrium eqn (1) the expression (17), we obtain

$$
\frac{d r}{d \varphi}-r \cdot\left(\frac{\sqrt{3}}{2} \cdot \frac{2-k}{k}+\frac{1}{2} \operatorname{tg} \varphi\right)=0
$$

The solution of this differential equation is a function

$$
r=A \exp \left(\frac{\sqrt{3}}{2} \cdot \frac{2-k}{k} \varphi\right) / \sqrt{\cos \varphi}
$$

The constants $A, \sigma_{0}$ and the parameters $\varphi_{a}, \varphi_{b}$ are defined in the general case numerically, using the boundary conditions (2). Substituting the stress $\sigma_{r}$ from (17) and the expression for the coordinate $r$ from (19) into the resolving eqn (1), after transformations we obtain an equation for determining the distribution of the modulus of elasticity $E(\varphi)$ :

$$
\frac{d E}{d \varphi}-\frac{2 \sqrt{3}}{k} \cdot \frac{\cos \varphi}{\sqrt{3} \sin \varphi+\cos \varphi} \cdot E=0
$$

After separation of variables in eqn (20) and integrating with the initial condition $\left(\varphi=\varphi_{a} ; E=E_{0}\right)$, we find the function $E(\varphi)$ :

$$
E(\varphi)=C \exp \left(\frac{\sqrt{3}}{2 k} \varphi\right)(\sqrt{3} \sin \varphi+\cos \varphi) \alpha
$$

where

$$
\alpha=\frac{3}{2 k} ; C=E_{0}\left[\exp \left(\frac{\sqrt{3}}{2 k} \varphi_{a}\right)\left(\sqrt{3} \sin \varphi_{a}+\cos \varphi_{a}\right)^{\alpha}\right]^{-1} .
$$

Given that the parameter $k$ depends on $v$ on the basis of expression (21), we can investigate the effect of Poisson's ratio on the character of the distribution 
function $E(r)$. As a result of the calculation solutions were obtained which differ from those shown in fig. 2 by no more than 5\%. Stress distributions in a nonhomogeneous structure for this theory of strength show similar curves to those in fig. 3.

This is not surprising since, as we know, the results of the strength of the maximum shear theory and the maximum-strain-energy theory in all problems of theory of elasticity do not give a contrast of more than $14 \%$.

Summarizing the results obtained in section 3.3, it is possible to draw the following conclusions. Creating equal stress thick-walled shells is possible by changing the modulus of elasticity of the material. Thus, the received solutions can be applied to materials for which it should be possible to make modifications of the modulus of elasticity of materials. Such materials include fiberglass, polymers and other composites.

\section{From equal-stress to equal-strength structures}

\subsection{Some mechanical properties of concrete}

In order to build a model equal-strength structure it is necessary that with a change of structure of the material its strength changes more slowly than the modulus of elasticity. An example would be modified cement concrete. Table 1 presents the main characteristics of this concrete [2]. Another example is polymer concrete filled silica flour [3] (see Table 2). It is evident that in these materials with a significant change in modulus of elasticity the strength varies slightly.

Table 1: $\quad$ Mechanical properties of concrete with the addition of microsilica.

\begin{tabular}{|c|c|c|c|}
\hline № & $\begin{array}{c}\text { Quantity microsilica in } \\
\text { the concrete mix, } \mathrm{kg} / \mathrm{m}^{3}\end{array}$ & $\begin{array}{c}\text { Prizm strength } \\
R_{b}, \mathrm{MPa}\end{array}$ & $\begin{array}{c}\text { Elasticity } \\
\text { modulus } \\
E_{b}, 10^{-4} \mathrm{MPa}\end{array}$ \\
\hline 1 & - & 42,5 & 3,62 \\
\hline 2 & 116 & 44,0 & 3,01 \\
\hline 3 & 142 & 38,0 & 2,16 \\
\hline
\end{tabular}

Table 2: $\quad$ Mechanical properties of polymer concrete filled silica flour.

\begin{tabular}{|c|c|c|c|}
\hline$№$ & $\begin{array}{c}\text { Level of filling } \\
\text { silica flour }\end{array}$ & $\begin{array}{c}\text { Prizm strength } \\
R_{b}, \mathrm{MPa}\end{array}$ & $\begin{array}{c}\text { Elasticity modulus } \\
E_{b}, 10^{-4} \mathrm{MPa}\end{array}$ \\
\hline 1 & - & 142 & 3,10 \\
\hline 2 & 50 & 146 & 4,50 \\
\hline 3 & 100 & 160 & 7,10 \\
\hline 4 & 200 & 148 & 10,5 \\
\hline 5 & 300 & 132 & 13,7 \\
\hline
\end{tabular}


For the correlation of strength and stiffness properties of the material the dependence $R=f(E)$ is introduced which allows agreement amongst themselves of the strength and deformation characteristics of the material. This function approximates the experimental data for the selected material. Using this dependence, we can solve the inverse problem to model the equal-strength structure. Nevertheless the structure may not be equal-stress, but the condition of equal-strength is ensured by the equality of the equivalent stress $\sigma_{0}$ at each point of the body to the strength of the material $R$ at this point.

\subsection{The inverse problem for an equal-strength cylinder}

This section describes the method of optimization of a cylindrical thick-walled shell of polymer-concrete based on the criterion of the strength of P. Balandin. This condition is confirmed experimentally for concrete in the field of all-round non-uniform compression [4]. The strength condition of P. Balandin in the coordinate system $\sigma_{1}, \sigma_{2}$ and $\sigma_{3}$ represents the surface of a paraboloid of revolution. Given the fact that the compressive stresses are contained within it with a minus sign, this expression is written as follows

$$
\sigma_{1}^{2}+\sigma_{2}^{2}+\sigma_{3}^{2}-\left(\sigma_{1} \sigma_{2}+\sigma_{2} \sigma_{3}+\sigma_{3} \sigma_{1}\right)+\left(R_{b}-R_{b t}\right)\left(\sigma_{1}+\sigma_{2}+\sigma_{3}\right)=R_{b} R_{b t},
$$

where $R_{b}$ is the design strength of concrete under axial compression (prism strength), and $R_{b t}$ is the design strength of concrete under axial tension. Since the concrete works poorly in tension it is possible to put $R_{b t}=0$ in (22). The application of this provision greatly simplifies the solution of the optimization problem. With this simplification expression (22) can be rewritten as:

$$
\sigma_{1}^{2}+\sigma_{2}^{2}+\sigma_{3}^{2}-\left(\sigma_{1} \sigma_{2}+\sigma_{2} \sigma_{3}+\sigma_{3} \sigma_{1}\right)+R_{b}\left(\sigma_{1}+\sigma_{2}+\sigma_{3}\right)=0
$$

In concrete, as follows from [5], in the limit state under the action of compressive stresses Poisson's ratio can reach values close to 0.5 . Therefore, for further calculations we take $v=0,5$. Assuming that the cylinder is in the plane strain conditions, the principal stresses can be determined as follows: $\sigma_{1}=\sigma_{z}=v\left(\sigma_{r}+\sigma_{\theta}\right), \sigma_{2}=\sigma_{r}$ and $\sigma_{3}=\sigma_{\theta}$. With this in mind, the strength condition (23) takes the form:

$$
0.75\left(\sigma_{r}\right)^{2}-1.5 \sigma_{r} \sigma_{\theta}+0.75\left(\sigma_{\theta}\right)^{2}+1.5 R_{b}\left(\sigma_{r}+\sigma_{\theta}\right)=0
$$

Eqn (24) describes a parabola in the implicit form, so the solutions need to be presented as a parametrical expression for the stresses $\sigma_{r}$ and $\sigma_{\theta}[6]$ :

$$
\sigma_{r}=-R_{b}\left(-0.5 \varphi+0.25 \varphi^{2}\right), \quad \sigma_{\theta}=-R_{b}\left(0.5 \varphi+0.25 \varphi^{2}\right)
$$

The relationship between the strength and the rigidity properties of the material is given by 


$$
R_{b}=\rho+\omega E_{b}
$$

where the coefficients $\rho$ and $\omega$ are determined on the basis of experimental data. In solving the problem, we use the condition that the Poisson's ratio $v=0,5$. From this it follows that in the plane strain condition

$$
\varepsilon_{r}=-\varepsilon_{\theta}
$$

Substituting (27) into the condition of compatibility of the strain components, we obtain the equation

$$
\frac{d \varepsilon_{\theta}}{d r}+2 \frac{\varepsilon_{\theta}}{r}=0
$$

The solution of eqn (28) is the expression

$$
\varepsilon_{\theta}=\frac{\varepsilon_{0}}{r^{2}}
$$

where $\varepsilon_{0}$ remains unknown.

To obtain the dependence of the modulus of elasticity of concrete $E_{b}$ from the radius we will use the expression for the strain $\varepsilon_{\theta}$

$$
\varepsilon_{\theta}=\frac{1}{E_{b}} \cdot\left[(1-v) \sigma_{\theta}-v \sigma_{r}\right]
$$

Substituting into (30) the value of Poisson's ratio $v=0.5$, and the expression (29), (25) and (26), after transformations we obtain

$$
E_{b}=\frac{\rho(1-2 \varphi) r^{3}(\varphi)}{4 \varepsilon_{0}-\omega(1-2 \varphi) \cdot r^{3}(\varphi)}
$$

Substituting (26) and (31) into eqns (17), we obtain expressions for the stresses in terms of the parameter $\varphi$

$$
\sigma_{r}=\frac{\rho \varepsilon_{0}\left(-5+12 \varphi-4 \varphi^{2}\right)}{3\left(4 \varepsilon_{0}-\omega(1-2 \varphi) \cdot r^{3}(\varphi)\right)}, \quad \sigma_{\theta}=\frac{\rho \varepsilon_{0}\left(1-4 \varphi^{2}\right)}{3\left(4 \varepsilon_{0}-\omega(1-2 \varphi) \cdot r^{3}(\varphi)\right)} .
$$

After substituting (32) in the equilibrium eqn (3) after transformations we obtain the nonlinear differential equation

$$
\frac{d r}{d \varphi}=\frac{2}{3} \cdot \frac{8 \varepsilon_{0}(3-2 \varphi) r-\omega(1-2 \varphi)^{2} r^{4}}{(1-2 \varphi)\left(16 \varepsilon_{0}+\omega(1-2 \varphi)^{2} r^{3}\right)}
$$

where $\varepsilon_{0}$ is a constant defined by the expression

$$
\varepsilon_{0}=\frac{R_{b}^{(0)}}{4 E_{b}^{(0)}}\left(1-2 \varphi_{a}\right) a^{3}
$$


Here $\varphi_{a}, R_{b}^{(0)}$ and $E_{b}^{(0)}$ are the initial (at $r=a$ ) values of the parameter $\varphi$, the design strength of concrete under axial compression and the modulus of elasticity of concrete. Equality (34) is obtained by substituting the following quantities into (30):

$$
\begin{gathered}
\nu=0.5, \varepsilon_{\theta}=\frac{\varepsilon_{0}}{a^{3}}, E_{b}=E_{b}^{(0)}, \\
\sigma_{r}=\frac{R_{b}^{(0)}}{12}\left(-5+12 \varphi_{a}-4 \varphi_{a}^{2}\right), \sigma_{\theta}=\frac{R_{b}^{(0)}}{12}\left(1-4 \varphi_{a}^{2}\right)
\end{gathered}
$$

To solve eqn (33) used the Runge-Kutta fourth order method.

\subsection{Calculation example}

Below, the solution for the thick-walled cylinder using the method given above on the basis of V. Paturoyev's experimental data [3] is presented.

The calculation was carried out using the following basic data: $E_{b}^{(0)}=3.1 \times 10^{4} \mathrm{MPa}, \quad R_{b}^{(0)}=141.0 \mathrm{MPa}, p_{a} / p_{b}=1.5, b / a=1.6, v=0.5 . \quad$ To determine the coefficients in the formula (26), we applied the standard mathematical functions of MathCAD 13, so therefore we obtained the following values: $\rho=126.7 \mathrm{MPa}$ and $\omega=4.6 \cdot 10^{-4}$.

For the first three lines of tab. 2. Fig. 4 shows the approximating dependence for polymer-concrete as well as the experimental points for which it is built.

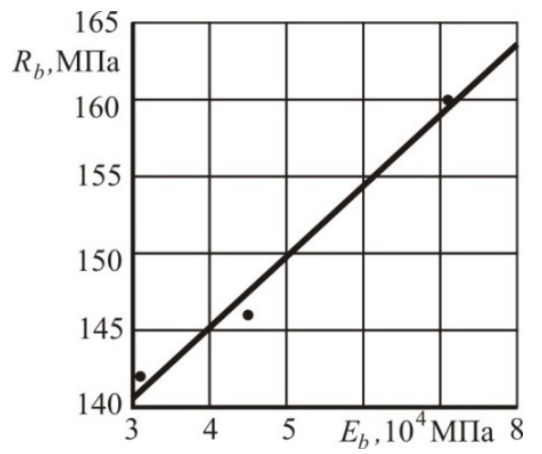

Figure 4: The relationship between strength and modulus of elasticity of polymer-concrete.

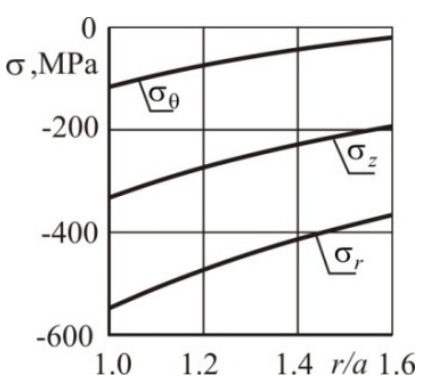

Figure 5: Stresses in an equalstrength polymer-concrete cylinder.

The solution of (33) is obtained by the Runge-Kutta fourth order method for the initial value of the argument $\varphi_{a}=-3.071$. The final value of the argument $\varphi_{b}=-2.222$. The values of the pressures on the cylinder are: $p_{a}=549.1 \mathrm{MPa}$ 
and $p_{b}=366.0 \mathrm{MPa}$ respectively. The value of pressure $p_{a}$ is found as follows. In the expression for the normal radial stress from (32) we substitute the values of the constants $\rho$ and $\omega$, the value of the parameter $\varphi=\varphi_{a}$, and also the value $r=a$. As a result we find $\sigma_{r}(r=a)=-549.1 \mathrm{MPa}$. Using the first of relations (2) we find the value $p_{a}$ specified above. The pressure $p_{b}$ equals $1.5 p_{a}$.

Stresses $\sigma_{r}, \sigma_{\theta}$ and $\sigma_{z}$ are shown in fig. 5. The resulting load in the equalstrength cylinder can be compared with the load for a homogeneous structure. By condition (24) the greatest equivalent stress is reached at an inner surface of the cylinder. For a homogeneous structure the value of the internal pressure $p_{a}^{\text {hom }}$ can be found by the formula

$$
p_{a}^{\text {hom }}=-\frac{2 R_{b}^{(0)}\left(s_{r}+s_{\theta}\right)}{\left(s_{r}-s_{\theta}\right)^{2}}
$$

where $s_{r}=-1$ and $R_{b}^{(0)}$ is the value of the design strength of polymer-concrete at the point where $r=a, s_{\theta}=\left(b^{2}+a^{2}-2 b^{2}\left(p_{b} / p_{a}\right)\right) /\left(b^{2}-a^{2}\right)$. The expression (36) is obtained as follows. We denote the stresses in the homogeneous cylinder as

$$
\sigma_{r}=p_{a}^{h o m} s_{r} ; \sigma_{\theta}=p_{a}^{h o m} s_{\theta}
$$

where functions $s_{r}$ and $s_{\theta}$ are obtained by solving the Lame problem for a thick-walled cylinder under boundary conditions

$$
r=a, s_{r}=-1 ; \quad r=b, \quad s_{\theta}=-d .
$$

Here $d$ is the ratio of the external pressure to the value of internal pressure. The design strength is assumed to be constant

$$
R_{b}=R_{b}^{(0)} \text {. }
$$

After substituting expressions (37) and (38) into eqn (24) we can find the pressure $p_{a}^{\text {hom }}$.

Calculation by formula (36) gives the pressure $p_{a}^{\text {hom }}=213.5 \mathrm{MPa}$. Above, we have determined the limit load for the equal-strength inhomogeneous structure $p_{a}^{i n h}=549.1 \mathrm{MPa}$. To determine the effect of the optimized model of the inhomogeneous equal-strength structure we introduce the efficiency ratio $\beta=p_{a}^{\text {inh }} / p_{a}^{\text {hom }}$, which shows how many times the external loads on the body can be increased, in comparison with the homogeneous analogue. For the equalstrength cylinder considered in this section $\beta=2.57$.

\subsection{From the model to the structure}

Creating a thick-walled cylinder where the modulus of elasticity varies along the radius by a continuous law is rather difficult. 
One way of solving the direct problems of the theory of elasticity of inhomogeneous bodies is to replace the continuous function $E(r)$ with a piecewise-homogeneous function. By analogy, the creation of optimized thickwalled cylinders consisting of several layers is proposed. Thus the modulus of elasticity of the material in each layer is determined by solving the inverse problem given above in which the continuous function $E(r)$ is defined.

The solution of the problem for the multilayered cylinder is rather simple. In each layer it corresponds to the solution of a Lame problem, and for definition of the constants the boundary conditions (2) and the conditions of ideal contact on boundaries between layers are used:

$$
u_{i}=u_{i+1} ; \quad \sigma_{r, i}=\sigma_{r, i+1}
$$

where $i$ is the layer number, $u$ is the radial displacement, and $\sigma_{r}$ is the radial stress. One of the fundamental questions is - how to choose the value of $E_{i}$ in the $i$-th layer. As shown in the analysis, in order to satisfy the condition of strength (23) at all points of the layer it is necessary that $E_{i}$ is equal to the value of $E(r)$ at the left edge of the layer.

Stresses $\sigma_{r}, \sigma_{\theta}$ and $\sigma_{z}$ for a three-layered cylinder are shown in fig. 6 and diagrams of equivalent stresses are given in fig. 7 .

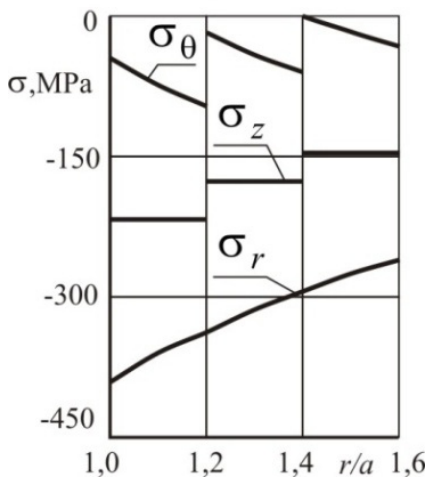

Figure 6: $\quad$ Stress distribution in a Figure 7: three-layer cylinder.

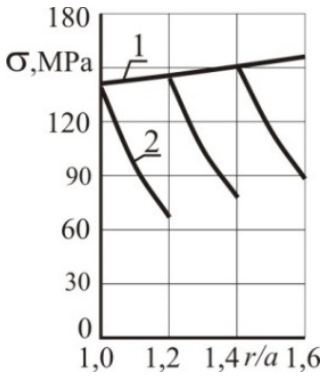

Strength $\quad R_{b} \quad(1)$ and equivalent stress (2) in a three-layer cylinder.

Let us compare the resulting load on the piecewise-homogeneous cylinder with a load for a homogeneous structure. Using (36), we can find the value of internal pressure: $p_{a}^{\text {hom }}=213.5 \mathrm{MPa}$. Comparing this pressure with the load for a piecewise-homogeneous cylinder $\left(p_{a}=p_{a}^{(3)}==-\sigma_{r}(r=a)=391.5 \mathrm{MPa}\right)$ we obtain the value of the coefficient effectiveness: $\beta=p_{a}^{(3)} / p_{a}^{\text {hom }}=1.83$. 
Similar calculations for the cylinder, consisting of four and five layers, give the following results: $\beta=p_{a}^{(4)} / p_{a}^{\text {hom }}=1.99, \beta=p_{a}^{(5)} / p_{a}^{\text {hom }}=2.09$.

It is obvious that with increasing number of layers in the cylinder the coefficient $\beta$ will be close to the value $\beta=2.57$, which corresponds to a model with continuous heterogeneity. Thus the developed multilayer structures can be called close to equal strength.

\section{Conclusions}

In this paper based on solving the inverse problem of elasticity theory of inhomogeneous bodies we have developed a method of changing the definition of the modulus of elasticity along the radius at which the equivalent stress in a thick-walled cylinder at each point is equal to the strength of the material. This cylinder is an equal-strength structure. Since in practice to create such a cylinder is hard enough, we propose the construction of multi-layer structures, in which the modulus of elasticity in each layer is determined by solving the inverse problem. This cylinder is close to being an equal-strength structure.

Generally, such shells may be formed from various materials (concrete or reinforced concrete, steels, polymers, etc.). Practical applications of these shells will be the result of collaborative work of specialists in mechanics, chemists and technologists.

\section{References}

[1] Andreev V.I., Nekotoryje zadachi i metody mehaniki neodnorodnyh tel, Izdat. ASV: Moscow, 2002.

[2] Batrakov V.G., Modificirovannyje betony. Teorija i praktika, 2 Issue: Moscow, 1998.

[3] Paturojev V.V., Polimerbeton, Strojizdat: Moscow, 1987.

[4] Genijev G.A. and Kissuk V.N., K voprosu obobshchenija teorii prochnosti betona. Beton i zhelezobeton, 2, pp. 16-19, 1965.

[5] Karpenko N.I., Obshchie modeli mehaniki zhelezobetona. Strojizdat: Moscow, 1996.

[6] Kamke E., Differentialgleichungen. Leipzig, 1959. 